\title{
Effect of display movement on tactile pattern perception
}

JAMES C. BLISS, STANFORD RESEARCH INSTITUTE AND STANFORD UNIVERSITY
HEWITT D. CRANE AND STEPHEN W. LINK, STANFORD RESEARCH INSTITUTE

The effect of display movement on the ability of subjects to recognize alphabetic shapes tactually was investigated. The display consisted of a computer-controlled 8-by-6 array of small airjet stimulators that could be physically translated in a small circle by means of a mechanical linkage. The experimental parameters were the stimulus duration, the angular velocity of the display, and the amplitude of the rotation. Recognition accuracy increased with stimulus duration between 100 and $400 \mathrm{msec}$. For a rotation amplitude of $0.8 \mathrm{~cm}$, a maximum in recognition accuracy occurred at a rotation velocity of $400 \mathrm{rpm}$, or $150 \mathrm{msec}$. per revolution. The optimum angular velocity appeared to decrease as the amplitude of rotation increased. From these results and certain related neurophysiological evidence, a hypothetical model is suggested which qualitatively can account for the data.

Bliss and Crane (1965) and Linvill and Bliss (1966) found considerable improvement in subject performance when tactually displayed letters were presented sequentially along a moving ticker-tape (Times Square) display rather than just a single stationary letter at a time. To study these tactile spatial-temporal interaction effects further, it was decided to test a different mode of stimulus pattern movement, namely a small circular translation (or nutation) of the entire display apparatus. In this mode, a single letter is presented in a fixed position on the display, and the display itself is translated about a circular locus, which is small compared to the size of the array. Each activated jet therefore follows a circular locus on the skin. By changing the diameter of the circular path and the velocity of the rotational motion, a fairly wide range of parametric conditions is obtained. Except for a slight increase in the total excited area because of the movement, the pattern remains fixed over the same anatomical position. Thus, a measure of performance with and without nutation over the same anatomical position can be readily obtained.

There were three reasons for the interest in this particular stimulus movement. First, it is reminiscent of vibrations in the eye, which are important for continuous vision. It is well known that if these eye vibrations are effectively cancelled, as in "stabilized image" experiments, vision rather quickly fades. Moreover, Krauskopf (1957), who introduced controlled motion in visual stabilized-image experiments, reported some improvement in acuity for oscillations at frequencies below $10 \mathrm{cps}$ and of sufficient amplitude.

Second, when lettering is read through a piece of shattered glass, where the average size of the intact glass is smaller than the size of the lettering, significant improvement can be achieved simply by vibrating the shattered glass in its own plane. In this way the distortion introduced by the fine structure of the shattered glass is averaged out. (Less improvement is obtained if the source material is vibrated instead of the glass.) For the tactile perception experiments, it was felt that since the dimensions of the overall tactile display are generally not more than a dozen or so two-point limen distances (i.e., close to the limit of spatial resolution on the skin), the effects of distortion introduced by nonuniform afferent receptor fields might similarly be averaged out by vibrating the pattern over the skin.

Third, some neurophysiological evidence suggests the probability of improved tactual perception with pattern vibration. Mountcastle (1957, p. 427), for example, from a study of cortical recordings observed: "It is a common observation quickly confirmed that tactile sensation is more acute if the exploring finger pad moves lightly over the test surface than if held motionless against it-for example, in differentiating fine grades of sandpaper, in the finger movements of the blind in reading Braille, or in assaying the quality of cloth. Oscillatory movement of the sensory receptor sheet will produce sharper peaks in the grid of cortical activity, with steeper gradients between them. Temporal alternation in the activity of two widely overlapped

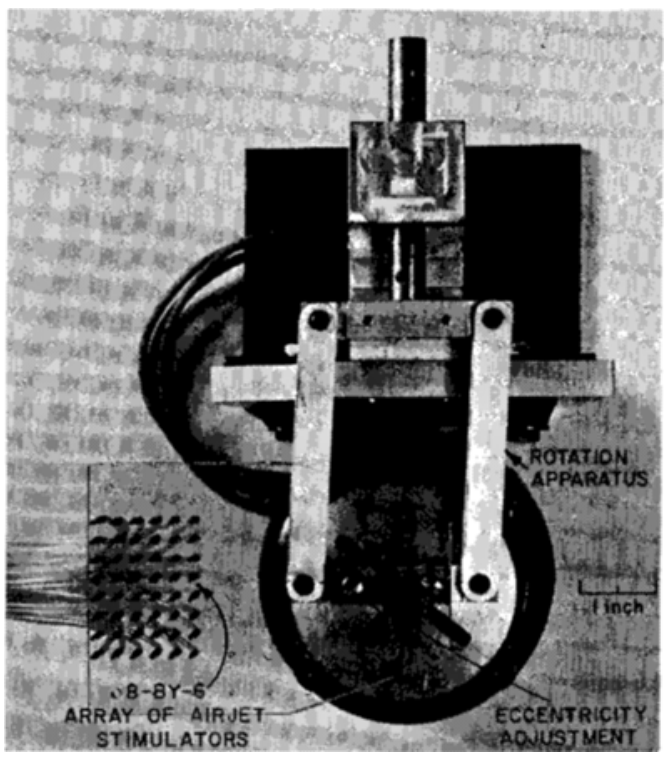

Fig. 1. Tactile stimulator apparatus (Top View). 


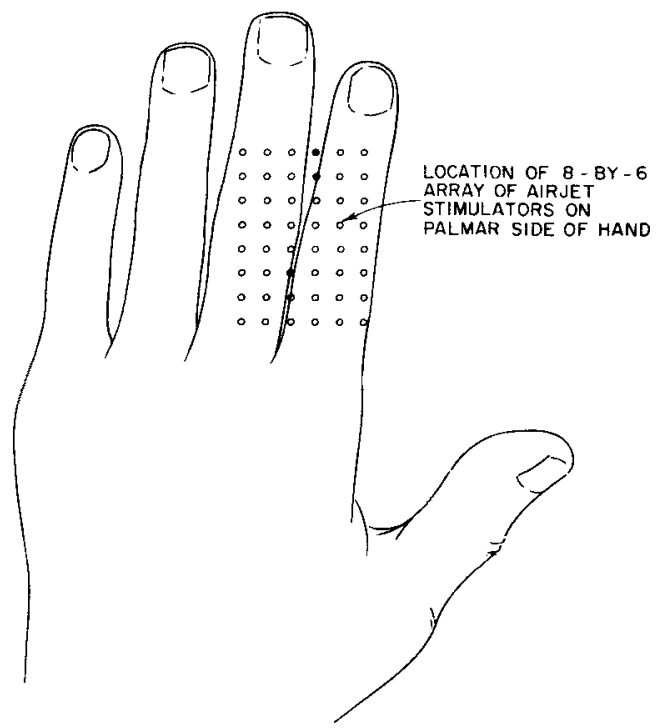

Fig. 2. Position of airjet array. The airjets were positioned about $1 / 8$ " below the palmar side of the hand.

groups of cells will accentuate the role of refractoriness of those cells common to both, rather than spatial facilitation, thus greatly steepening the gradients of activity between the two peaks." Three different experiments are reported here:

(a) In the first experiment, static and "rotated" patterns were presented in alternate sessions, with various values of the stimulus duration, interstimulus interval, and angular velocity of rotation. The quantitative results clearly indicate better performance with the rotating display.

(b) The results from the first experiment were sufficiently positive that a second experiment was performed to study more precisely the effects of overall stimulus duration and pattern rotation velocity. In this experiment the subject was self-paced, since results from the first experiment indicated that a forced re-
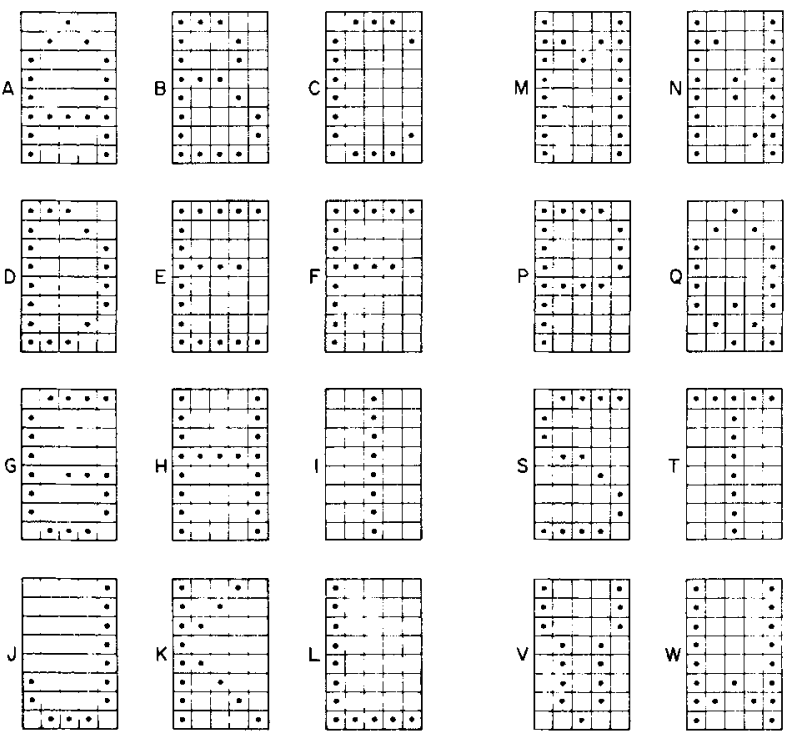

sponse interval caused errors unrelated to the parameters of interest.

(c) In the third experiment, the effect of rotational amplitude was studied.

\section{Apparatus and Procedure}

The experiments were carried out under control of a computer system described elsewhere by Bliss and Crane (1964). In this system a CDC 8090 Computer is used to store stimulus patterns and to control the sequence in which the patterns are presented. For each frame of presentation the computer transmits a sequence of eight 12-bit words (each word representing one row of the spatial pattern to be displayed) to specially constructed external equipment. The external equipment stores up to 96 bits ( 8 words) and activates the specified tactile stimulators in $8 \mathrm{msec}$. In the experiments reported here, only half the array, 8 by 6 , was used. The basic tactile stimulator generates bursts of air from an $0.031-i n$. outlet port, under control of a sensitive high-speed electromagnet. The pulse pressure, measured $1 / 8 \mathrm{in}$. above the airjet outlet, is about $3 \mathrm{psi}$, with a rise and fall time of about a millisecond and an overall pulse width of about $2.5 \mathrm{msec}$. A 200-cps pulse repetition rate was used throughout the experiments, implying that the airjet is turned on and off 20 times, for example, during a stimulus duration of $100 \mathrm{msec}$.

The advantages of airjet stimulators a re that relatively uniform stimulation is produced over nonuniform cutaneous surfaces and that stimulator spacing can be easily changed. The stimulator array used is shown in Fig. 1, and the location of the stimulators with respect to the palmar side of the hand is shown in Fig. 2. The subjects suspended the fingers of the right hand over the matrix, with the arm and palm of the hand supported by a rest.

Inexperienced or "tactually naive" subjects initially have considerable difficulty reading a simple capital
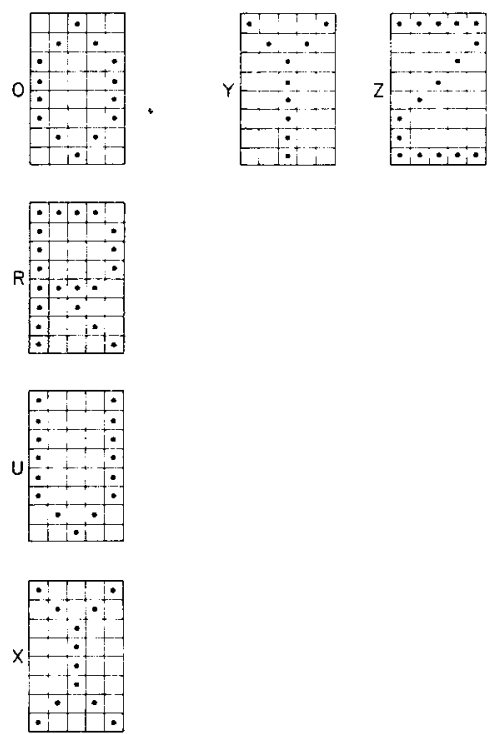

Fig. 3. Block letter alphabet. 


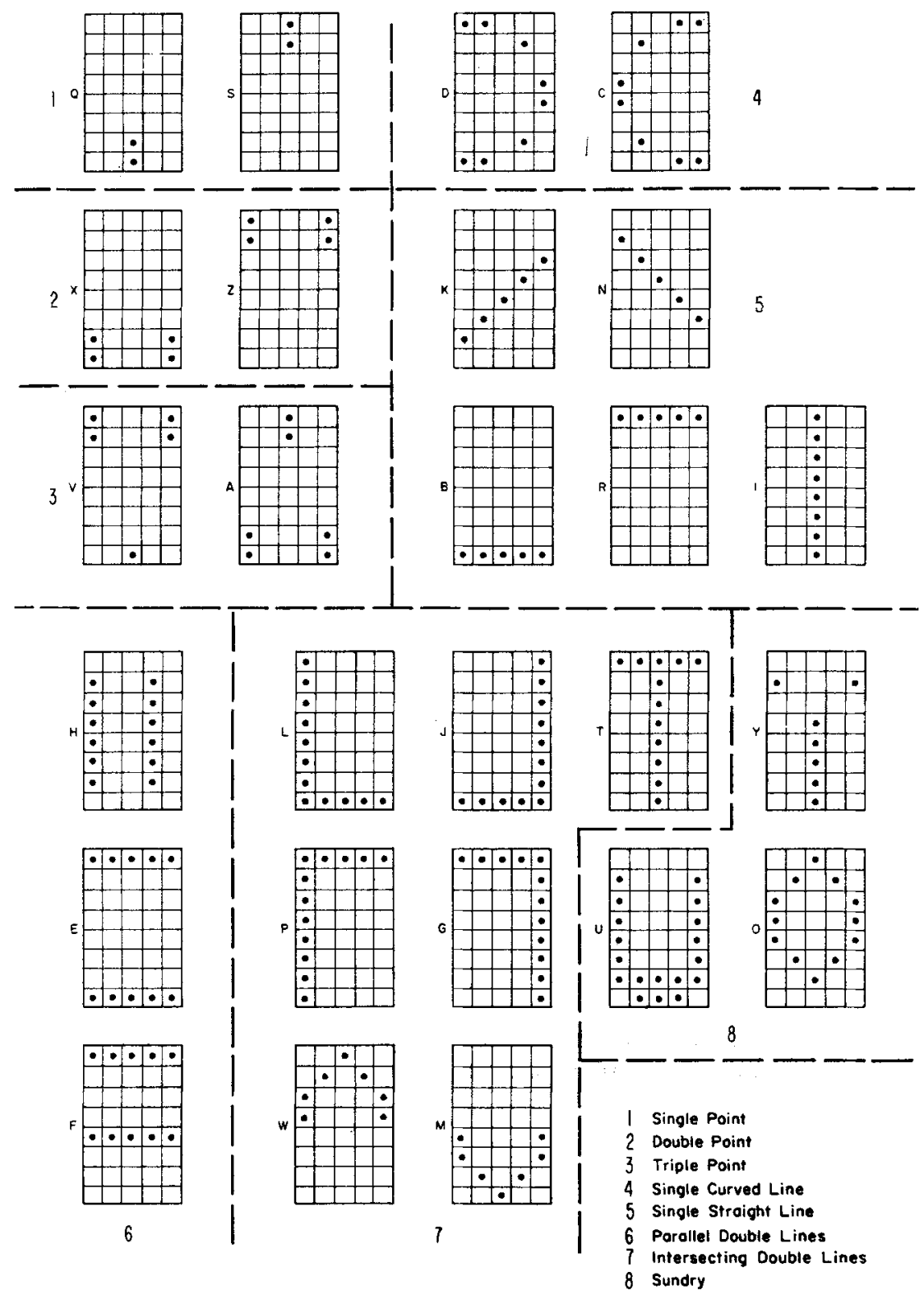

Fig. 4. Experimentally developed alphabet.

letter alphabet of the form shown in Fig. 3. The general reaction is that there is just too much air; each letter seems to feel like a blast of air without much structure. For this reason the more abstracted alphabet forms of Fig. 4 were developed. The procedure for designing this alphabet was subjective and based on trial and error methods. To take advantage of possible positive effects from the transfer of visual imagery to tactile imagery, a progressive process of modifying the block letters was pursued, and an attempt was made to maintain as much similarity to the standard letters as possible. In many cases this was possible, but in others, unrelated symbols were substituted to facilitate rapid discrimination. Letter modifications continued until each letter could be recognized with almost perfect accuracy when the letters were presented in random order in a fixed (nonmoving) position for about 150-msec. duration and with an interstimulus interval of approximately $1 \mathrm{sec}$.

The specially designed alphabetic symbols of Fig. 4 were used in the first two experiments reported here; the block letters of Fig. 3 were used in the third experiment.

Subject $S_{1}$ was trained in a series of 1-hr. sessions in which the stimulus duration was $300 \mathrm{msec}$. and the interval between stimuli was progressively decreased from $1.5 \mathrm{sec}$. to $0.6 \mathrm{sec}$. Display rotation was introduced in alternate double sessions as shown in Fig. 5. For these sessions, the angular velocity was $870 \mathrm{rpm}$ and the amplitude of rotation was $0.8 \mathrm{~cm}$. Figure 5 indicates that even during training, display movement improved performance. By the 13th session the subject had reached the asymptote of his performance. 


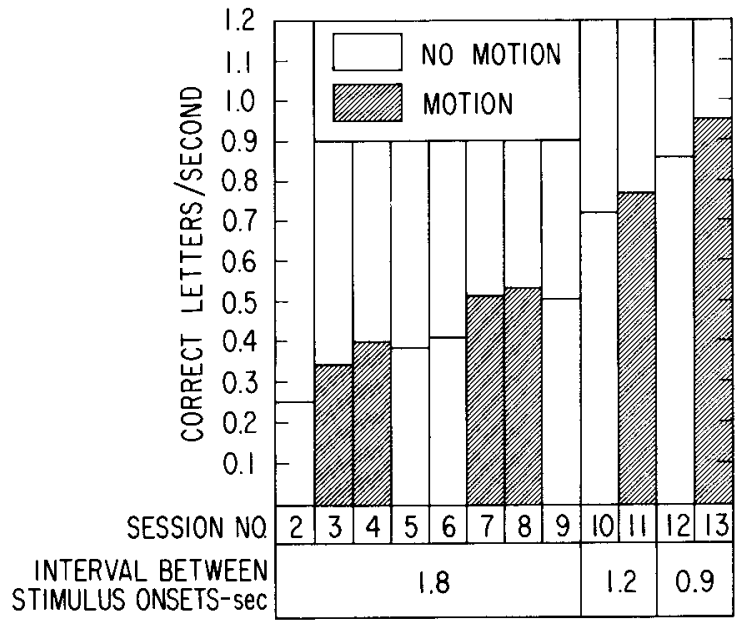

Fig. 5. Performance during training for $s_{1}$. Stimulus duration was $0.3 \mathrm{sec}$, and the rotation velocity was $870 \mathrm{rpm}$.

\section{Experiment 1. Performance With and Without Stimulus Pattern Motion}

This, experiment helped to determine under what stimulus conditions nutation of the stimulus apparatus improved a subject's performance. Two factors were investigated: (a) motion versus nonmotion of the stimulating apparatus, and (b) variation of the total number of display revolutions per presentation, accomplished by varying the stimulus presentation time.

The rotation frequency and stimulus duration for each session is shown in Fig. 6, along with the performance scores. These results are for Subject $S_{1}$, who was highly practiced in making verbal responses to briefly presented tactile stimuli. In each trial of this experiment, a letter was presented to the subject, and 900 msec. after the onset of the stimulus, a new stimulus was presented. The fixed trial time required the subject to respond faster when stimulus duration was increased. During each experimental session, presentation time, and hence the number of revolutions per presentation, was held constant; but presentation time was varied from session to session over a range of $\mathbf{5 0}$ to 450 msec. Rotation speeds of 435,870 , and 1250 rpm were used. Each session consisted of four test runs of approximately 90 trials each. The correct letters per second from the 12 sessions, 36 to 43,48 , and 50 to 53 ,

Table 1. Analys is of Variance for Sessions 36-53

\begin{tabular}{lrrrc} 
Source & $\begin{array}{c}\text { Sums of } \\
\text { Squares }\end{array}$ & df & $\begin{array}{c}\text { Mean } \\
\text { Square }\end{array}$ & $\begin{array}{c}\text { Level of } \\
\text { Significance }\end{array}$ \\
\hline Motion vs. Nonmotion & 78.65 & 1 & 78.65 & $a<0.005$ \\
Sessions & 96.35 & 5 & 19.27 & $a=0.010$ \\
Interaction & 73.25 & 5 & 14.65 & $a<0.050$ \\
Error & 187.28 & 36 & 5.20 & \\
Total & 435.53 & 47 & & \\
\hline
\end{tabular}

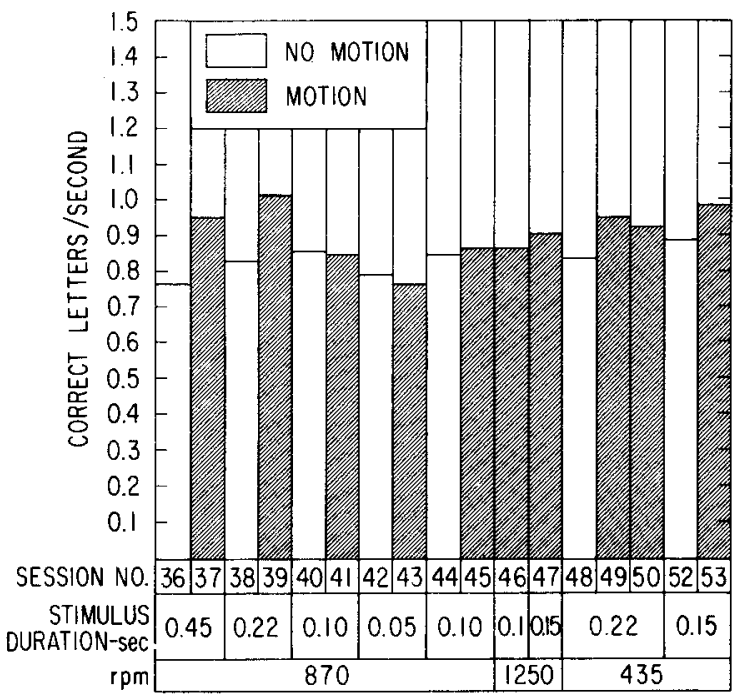

Fig. 6. Average test scores for each session. The interval between stimulus onsets was 0.9 sec.

were analyzed according to a two-way analysis of variance. The results of this analysis are shown in Table 1.

Considering the order of magnitude of the interactions (Table 1), it cannot be concluded that the component due to interaction is insignificant. However, it may be safely concluded that averaged over revolutions per letter, there is a significant $(\alpha<0.005)$ difference between the motion versus nonmotion methods of stimulus presentation, motion resulting in a marked increase in the number of correct responses by the subject.

As shown in Fig. 6, for each session a different response time and a different number of revolutions per letter of the airjet array were used. It can be concluded $(\alpha<0.01)$ that there are significant differences among the six pairs of sessions.

An analysis was made of the frequency of occurrence of error-error pairs on successive trials during a session. The purpose was to determine whether the paced response resulted in a greater number of errors, owing to the decrease in time allowed to respond with decreased interstimulus interval. It was found that error-error pairs increased with presentation time and hence, increased with reduced response time, assuming that the response time started after the stimulus was turned off. In summary, Experiment 1 indicated:

(a) Motion (versus nonmotion) of the tactile pattern results in a substantial increase in the number of correct responses.

(b) Limiting the time to respond resulted in increased errors.

These results suggested more careful control of the presentation factors as well as introduction of a selfpaced stimulus presentation rate. 
Table 2. Data from Stimulus Movement Experiment 2 for $s_{1}$ and $s_{2}$ [Entries are Means of Number Correct (out of a possible 234) from Three Tests Averaged over $s_{1}$ and $\left.s_{2}\right]$

\begin{tabular}{ccccccc} 
Factor 1 & \multicolumn{5}{c}{ Foctor J (rpm) } & \\
\cline { 2 - 6 } (msec) & 0 & 200 & 400 & 800 & 1200 & Row Mean s \\
\hline 100 & 212 & 208 & 227 & 227 & 216 & 215 \\
200 & 210 & 226 & 232 & 227 & 227 & 224 \\
300 & 214 & 225 & 222 & 227 & 228 & 223 \\
400 & 220 & 230 & 230 & 227 & 224 & 226 \\
Column Means & 214 & 222 & 228 & 223 & 224 & $222=$ \\
& & & & & & overall mean \\
\hline
\end{tabular}

Experiment 2. Effect of Stimulus Pattern Rotation Velocity and Stimulus Duration Time

In order to examine more carefully the influence of stimulus presentation time and the rotation velocity of the display, a complete factorial experiment was designed. Of the two male subjects and one female subject participating in the experiment, $\mathrm{S}_{1}$ had extensive prior training in tactile perception and $S_{2}$ and $S_{3}$ were given $9 \mathrm{hr}$. of prior training. Each subject's task was to identify a letter presented tactually, make a verbal response, and then depress a foot switch to signal the computer to proceed with the next stimulus presentation. Each experimental session consisted of a control test followed by three tests under a new experimental condition. During both control and experimental tests, subjects were presented the alphabet of 26 letters three times (i.e., a total of 78 letters) in a random order.

Two factors, consisting of four levels of presentation time and five levels of rotation velocity, were replicated by each subject. Factor I, presentation time, consisted of levels $100,200,300$, and $400 \mathrm{msec}$., while factor $\mathrm{J}$, rotation velocity, consisted of levels $0,200,400,800$, and $1200 \mathrm{rpm}$. At the beginning of each control session a test was run at $400 \mathrm{msec}$. and $800 \mathrm{rpm}$.

The data for each subject were corrected for guessing using the formula

$$
p=\frac{P(c)-g}{1-g}
$$

where

$P(c)=$ observed proportion of correct responses $\mathrm{p}=$ probability of a correct response without guessing

$\mathrm{g}=$ probability of guessing correctly.

The only substantial changes in the data due to this correction were for subject $s_{3}$, who performed very poorly throughout the experiment. In spite of several additional hours of training, $s_{3}$ averaged less than half the number of correct responses recorded for either $\mathrm{S}_{1}$ or $\mathrm{S}_{2}$. Because of this rather poor performance, the data for $\mathrm{S}_{3}$ were analyzed to obtain only information concerning the effects of motion versus nonmotion of the tactile display. The average number of correct responses (out of a possible 234) was 76.7 in the non-

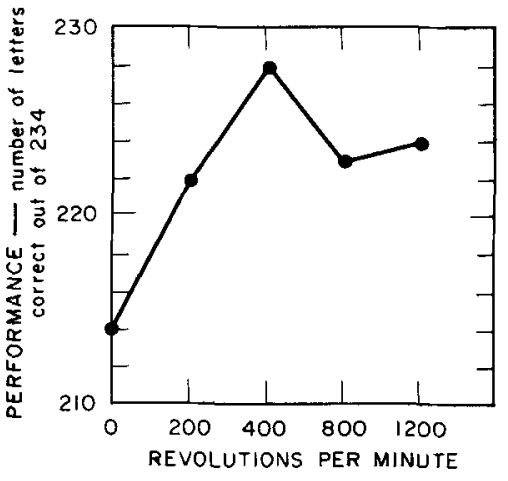

Fig. 7. Performance as a function of RPM.

motion con lition and 89.4 in the motion condition. A simple analysis of variance showed that the effect of motion contributed substantially toward improved performance $(\alpha<0.001)$.

The results for $S_{1}$ and $S_{2}$ are reported in Tables 2 and 3 . Since the control variable contained little variation, it was not included in the analysis. Figures 7 and 8 show the performance versus "treatment."

The data in Fig. 7 show that for the range of stimulus durations explored, a maximum rate of performance occurred at $400 \mathrm{rpm}$, corresponding to a rotation period of $150 \mathrm{msec}$. Figure 8 shows improved performance with increasing stimulus presentation time. (Subject $S_{1}$, who was highly trained in tactual perception, showed a strictly linear increase in performance as a function of presentation time. The deviation from linearity shown in Fig. 8 resulted from the performance of subject $S_{2}$, who was not as highly trained as $S_{1}$.) Viewing the maximum row and column means in Table 2 , it is apparent that a maximum in performance occurred at $400-\mathrm{msec}$. presentation time and $400 \mathrm{rpm}$. These conditions correspond to $2-2 / 3$ complete revolutions of the display during each stimulus presentation, with 150/5, or 30 , separate presentations of the pattern per rotation, spaced $360 / 30$, or 12 degrees apart along the circumference.

\section{Experiment 3. Effect of Amplitude of Stimulus Pattern Move-} ment

In this experiment the effect of rotation amplitude was investigated. In order to lower the percentage of accuracies below those of the previous experiment so that improvements would stand out more clearly, and also to reduce the effects of previous training, it was

Table 3. Analysis of Variance for Experiment 3

\begin{tabular}{lrccc} 
Source & df & $\begin{array}{l}\text { Sums of } \\
\text { Squares }\end{array}$ & $\begin{array}{l}\text { M } \\
\text { Mean Square }\end{array}$ & $\begin{array}{c}\text { Level of } \\
\text { Significance }\end{array}$ \\
\hline Rows & 3 & 753.58 & 251.19 & $a<0.05$ \\
Columns & 4 & 812.70 & 203.18 & $a<0.05$ \\
Interaction & 12 & 597.85 & 49.82 & n.s. \\
Error & 20 & 857.40 & 42.87 & \\
Total & 39 & 3021.53 & & \\
\hline
\end{tabular}




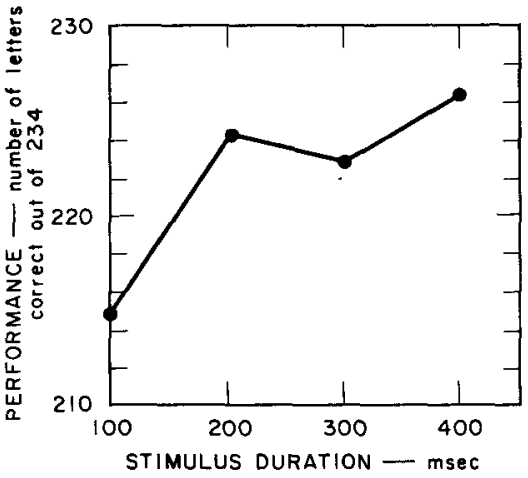

Fig. 8. Performance as a function of stimulus presentation time.

decided to run this test using the more difficult blockletter alphabet of Fig. 3, rather than the specially designed alphabet used in the previous experiment.

Subject $\mathrm{S}_{1}$ was given $2 \mathrm{hr}$. of training on the blockletter alphabet, which he had never felt before. Then he was given a series of tests with different amplitude settings. Four tests were run for each amplitude setting. In Fig. 9 are plotted the results for each setting, the spread in results for the four tests, and the average. The numbers next to the plotted points indicate the sequence of these tests. The sequence is significant because the subject was not very well trained on this alphabet before starting the experiments, and therefore some learning during the sequence was expected. The relatively small extent of this learning can be seen from the slightly increased performance between the first and last experiment for the same conditions, namely $0.8 \mathrm{~cm}$ and $400 \mathrm{rpm}$.

\section{Hypothetical Model}

It is clear from the results described here that certain amplitude and rotation velocities improve performance. Subjects commented on the sharpened and enhanced sense of perception in these ranges of quantitative improvement. Subjects' reports also indicate that the "best" frequency of rotation tends to decrease monotonically as the amplitude of rotation increases, sug-

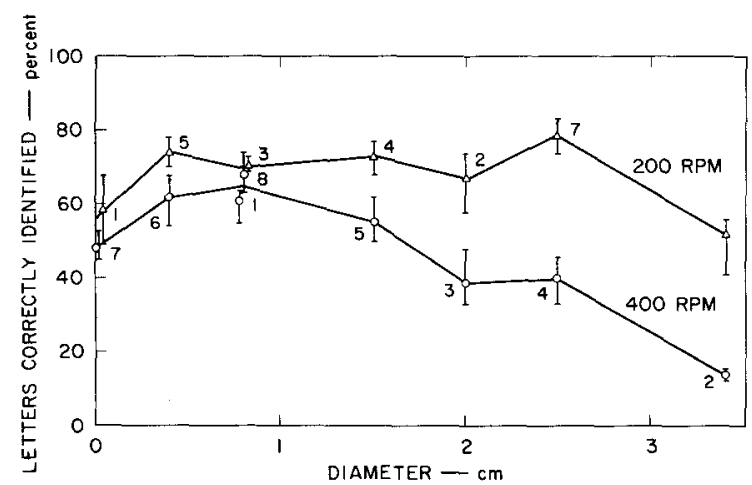

Fig. 9. Effect of amplitude of circular translation of stimulus array on recognition of block letters. gesting that best performance may be obtained for a certain linear velocity of the display. What this velocity might be can be estimated from the second and third experiments. For an amplitude of about $0.8 \mathrm{~cm}$, a peak in performance for $S_{1}$ and $S_{2}$ was obtained with a display rotation frequency of $400 \mathrm{rpm}$ (or $150 \mathrm{msec}$. per revolution), leading to a velocity along the circular locus of about $15 \mathrm{~cm} / \mathrm{sec}$. (This velocity corresponds to a moving-belt display of 67 words per minute, reported in Linvill and Bliss, 1966.)

A possible neurological mechanism underlying these results is suggested by Mountcastle (1957), who found that in response to each point stimulus at the periphery (say, point A in Fig. 10) there appears a corresponding point $A^{\prime}$ of maximum excitation at the cortex, with lesser magnitudes of excitation surrounding this central point, and a circular band of inhibition even further removed from the center. Of particular importance is a monotonic increase in latency of response as the recording point moves from the strong central point toward the inhibitory surround. In terms of such observations, the results of this paper might be interpreted according to the following model.

Visualize, for each point stimulus at the periphery, a "wave" of activity propagating from a central point at the surface of the cortex, as suggested in Fig. 11, excitation becoming weaker with further and further distance until a zone of inhibition is reached. Consider now a second peripheral location $B$ with corresponding central point $B^{\prime}$. Let point $B$ be stimulated $T$ seconds after point $A$. If the interval $T$ is very short, then excitation appears at points $A^{\prime}$ and $B^{\prime}$ essentially simultaneously, and similar waves propagate subsequently from each of these points. If $T$ is very long, the effects of the wave from A are substantially dissipated before excitation of $B$, and the subsequent course of excitation from $B^{\prime}$ resembles that from $A^{\prime}$ except for a shift in position and time. For an intermediate range of $T$ values, excitation at $B^{\prime}$ tends to occur during the interval of inhibition from $A^{\prime}$. In this case, a significant decrease, or even complete elimination, of the excitatory response at $B^{\prime}$ could be expected. At points inter-

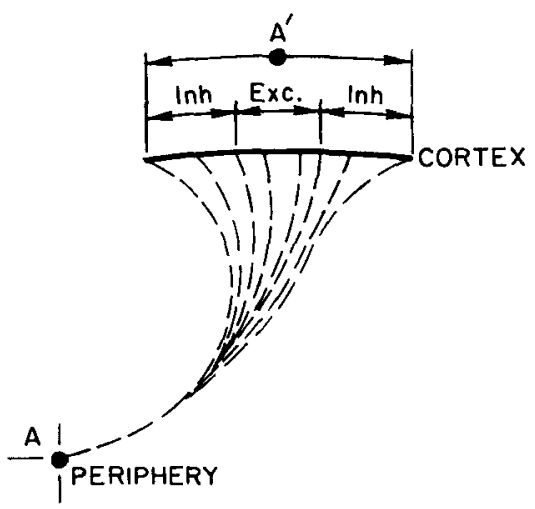

Fig. 10. Cortical response to a peripheral point stimulus. 


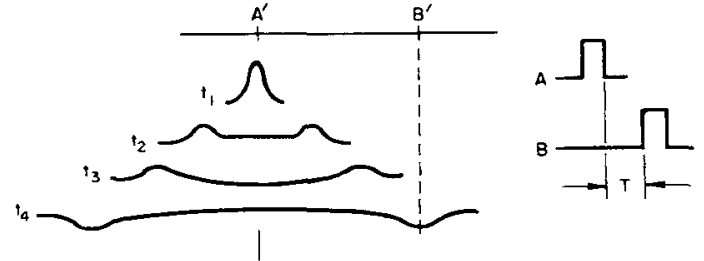

Fig. 11. Traveling wave from the maximum point of excitation, $\mathbf{A}^{*}$, in response to stimulation at peripheral Point $\mathbf{A}$.

mediate between $A^{\prime}$ and $B^{\prime}$, however, a summation of excitation from $A^{\prime}$ and $B^{\prime}$ would be possible.

A subsequent excitation at $A$, following the excitation at $A$ and then $B$, involves similar considerations. Thus, with repetitive stimulation, ..., A, B, A, B, . . one could expect to find a rate of excitation, of period $2 \mathrm{~T}$, at which the net sensation is strongest at an intermediate position between two even relatively widely spaced points $A$ and $B$. These arguments can be extended to consideration of an airjet stimulator traveling in a circular locus, where the pulsating air causes excitation at point a', then $b^{\prime}$, then $c^{\prime}$, and so forth as in Fig. 12. In the critical frequency range, we visualize the excitation summing at the central region, with the peripheral responses diminished by inhibition.

Of particular interest with regard to this interpretation of cortical events is the experimental finding that the optimum frequency of rotation tends to decrease inversely with the diameter of the rotation. In terms of the two-point arrangement of Fig. 11, if A and B were separated further so that the inhibition wave took correspondingly longer to become effective in the region of the other point, then for the optimal cancellation of excitation, interval $T$ would have to be correspondingly increased. Similarly, if the radius of rotation were doubled, then, as a first approximation, the optimum time for the stimulator to arrive at its diametrically opposite location would be doubled-or, the optimum rotation frequency would be halved.

Because the stimulus pattern with the rotating display is complex (due to simultaneous excitation of many rotating jets), a simple qualitative experiment was performed using a single jet traveling in a circle over the tip of the index finger. The prediction based on this simple model was that at very low rotation frequency, the subject would mentally be able to follow the slowly moving path of the stimulator. At a very high rotation frequency, the subject would have the sensation that a sharp circular rim was pressing into his skin, since, at high rotation frequencies, all parts of the path are essentially excited simultaneously. One might think that there would be a relatively smooth transition from the slow-speed sensation to that of the solid rim; however, the arguments in connection with Fig. 12 predict an intermediate sensation at which there is primarily a strong central sensation with perhaps little, if any, sensation of rotation. An intermediate sensation of this form is indeed found, and at a rotation rate close to that found to be optimum for the complex display patterns. However, the sensations are by no means geometrically perfect. Below are some verbatim responses of subjects as the rpm was progressively increased:

(a) At very low rpm: "... feel point moving around but not a good circular feeling-perhaps more elliptical, or if finger is not positioned accurately only perhaps half an arc."

(b) At higher rpm: ". . begin to feel more of a closed locus but again more elliptical with axis changing randomly, although there seemed to be a preferred direction. Sometimes felt circular." (c) Still higher: "There seemed to be a zone in which the feeling was more like a solid disk of air with some edge modulation-like a coin settling down on a table." (Note: The sensation still remained as a single central zone even with the finger moving about.)

(d) At high rpm: "... felt like a rim of air, especially if the finger moved around; in an exploring manner."

\section{Some Relevant Neurophysiological Data}

Such a mechanistic model leads to a search for inhibitory phenomena involving interaction over periods of the order of a $100 \mathrm{msec}$. or so. It would indeed be interesting to obtain physiological recording data with a stimulator array moved over the skin, or even a point stimulus rotating over the skin, in the manner discussed. However, data are generally available only for a single fixed stimulator. Even so, there does appear to be scattered data indicating such periods of inhibitory effects. Below is a sampling of such evidence.

A relevant study is that of Towe and Amassian (1958), in which the digits of rhesus monkeys were stimulated electrically and single-unit responses were recorded in the somatosensory cortex. It was found that the discharge of 40 of the 110 units encountered could be prevented by prior or simultaneous stimulation at a nearby point, even when this nearby point stimulated alone would not itself cause the unit to fire. This inhibition

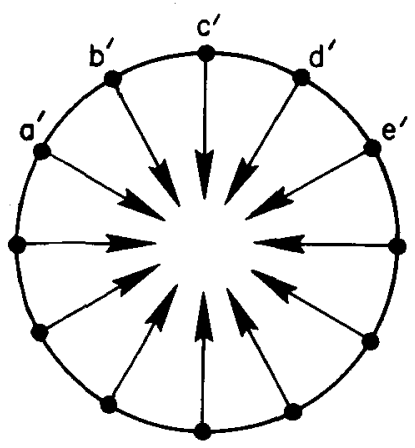

Fig. 12. Excitation of the cortex in response to a circular locus of stimulation at the periphery. 
lasted about $80 \mathrm{msec}$. and was followed by a period of slight facilitation. The duration of this inhibitory effect was directly proportional to the intensity of the testing stimulus and the spatial separation of the conditioning and testing stimuli.

Phenomena found in the components of compound evoked potentials recorded from the scalp of humans suggest similar inhibitory effects, lasting about 100 msec., followed by facilitation (Uttal and Cook, 1964, and Allison, 1962).

Related phenomena have also been noted on a peripheral level. Lindblom (1965) found long-duration inhibition phenomena in dorsal root units of monkeys as a result of mechanical stimulation of the distal glabrous skin. By means of threshold studies, he demonstrated that each impulse is followed by a relative refractory period that lasts more than $100 \mathrm{msec}$. Repetitive discharge delayed the recovery further and produced a cumulative increase in threshold which rendered maintained firing at frequencies above about 60 impulses per second difficult or impossible.

In addition, Uttal (1966) reports an amplitude oscillation in the compound evoked potentials recorded at the superficial point (i.e., the point closest to the surface of the skin) of the ulnar nerve above the elbow. Pulse electriçal stimuli were applied to the superficial point of this nerve at the wrist. The magnitude of the oscillation was found to depend on stimulus intensity and interstimulus interval, although the period of the oscillation was very nearly constant at about $100 \mathrm{msec}$.

While these results are suggestive, more definitive data are needed before the physiological mechanisms underlying the behavioral results are clearly understood. From a practical standpoint, however, spatial pattern rotation appears to produce effects which may be important for tactile communication systems.

\section{References}

Allison, T. Recovery functions of somatosensory evoked responses in man. EEG clin. Neurophysiol., 1962, 14, 331-43.

Rliss, J. C., \& Crane, H. D. A computer-aided instrumentation system for studies in tactual perception. Proc. 16th Ann. NAECON Conf., Dayton, Ohio, 1964; 375-384.

Bliss, J. C., \& Crane, H. D. Experiments in tactual perception. NASA Contractor Report NASA CR-322, November 1965, Prepared by Stanford Research Institute, Menlo Park, California under Contract NAS 2-1679.

Krauskopf, J. Effect of retinal image motion on contrast thresholds for maintained vision. J. Opt. Soc. Amer., 1957, 47, 740-744

Lindblom, V. Properties of touch receptors in distal glabrous skin of the monkey. J. Neurophysiol., 1965, 128,966-985.

Linvill, J. G., \& Bliss, J. C. A direct translation reading aid for the blind. Proc. of IEEE, 1966, 54, 40-51.

Mountcastle, V. B. Modality and topographic properties of single neurons of cat's somatic sensory cortex. J. Neurophysiol., 1957, $20,408-434$.

Towe, A. L., \& Amassian, V. E. Patterns of activity in single cortical units following stimulation of the digits in monkeys. $J$. Neurophysiol., 1958, 21, 292-311.

Uttal, W. R. Do compound evoked potentials reflect psychological codes? Psychol. Bull., 1965, 64; 377-392.

Uttal, W. R., \& Cook, L. Systematics of the evoked somatosensory cortical potential: a psychophysical-electrophysiological comparison. Ann. N. Y. Acad. Sci., 1964; 112 (Art. 1), 60-80.

\section{Note}

1. The work reported in this paper was supported by the Research and Technology Division of the Air Force Avionics Laboratory, Aeronautical Systems Division, under Contract AF 33(615)-1099 with Stanford Research Institute. The airjet tactile stimulators used in this study were developed at Stanford Research Institute under Contract NAS 2-1679 with Ames Research Center, National Aeronautics and Space Administration, Moffett Field, California.

(Received in the Editorial Office May 14, 1966.) 